\title{
Value of PET-CT Staging in Lymphoma Patients at Baseline over Clinical Staging
}

\author{
Tasnim Ahmed, Fatima Begum and Shamim M F Begum \\ National Institute of Nuclear Medicine and Allied Sciences (NINMAS), Dhaka, Bangladesh \\ Correspondence Address: Dr. Tasnim Ahmed, Senior Medical Officer, National Institute of Nuclear Medicine and Allied Sciences, \\ Block-D, 7th-10th floor, BSMMU Campus, Dhaka-1000, Bangladesh, Email: dr.synthi@gmail.com
}

\begin{abstract}
Objective: Lymphoma is the cancer of lymphatic system. Treatment strategy of lymphoma depends upon the staging of disease. Accurate staging can guide the required regimen and can minimize the therapeutic toxic effect and reduces the morbidity and mortality associated with over treatment. The purpose of this study was to observe the upstaging or down staging of lymphoma patients at baseline by performing PET-CT imaging over clinical staging who were referred to National Institute of Nuclear Medicine \& Allied Sciences (NINMAS) for baseline staging.

Patients and Methods: This cross sectional observational type of study was carried out in NINMAS from July 2017 to June 2018. A total of 26 newly diagnosed lymphoma patients referred to NINMAS for PET-CT scan for baseline staging were included in this study.

Results: Out of the 26 patients (14male and 12 female; mean age: $43.42 \pm$ 17.98 years), 14(53.8\%) patients were diagnosed with Hodgkin Lymphoma and $12(46.2 \%)$ with Non Hodgkin Lymphoma. In this study, 14(53\%) patients were upstaged in PET-CT scan based staging in comparison to clinical staging. Key lesion was assessed by SUVmax in PET-CT scan of different stages of lymphoma. Highest key lesion was found in stage IV. Out of 26 patients, 7(27\%) showed extranodal involvement in bone (3 lesions), liver (3 lesions), lung (1 lesion) and spleen (1 lesion).

Conclusion: This study was undertaken to assess the role of FDG PET-CT scan for staging of lymphoma patient at baseline. In this study, 53\% of the patients were upstaged in PET-CT scan based staging in comparison to clinical staging. PET-CT plays an important role for accurate staging in lymphoma patients at baseline which increases the accuracy of subsequent response assessment.

Key words:PET-CT, Lymphoma.
\end{abstract}

Bangladesh J. Nucl. Med. Vol. 22 No. 1 January 2019

Doi: https://doi.org/10.3329/bjnm.v22i1.40498

\section{INTRODUCTION}

Lymphoma is a heterogeneous group of malignant neoplasm of the reticuloendothelial system, which may be subdivided broadly into Hodgkin lymphoma (HL) and Non Hodgkin lymphoma (NHL). In a study, prevalence of NHL is $16.9 \%$ and $\mathrm{HL}$ is $3.9 \%$ among all hematological malignancies in Bangladesh (1).NHL is more common which represents $85 \%$ of all lymphoma. HL is subdivided into classical and non-classical type. Non-classical type represents nodular lymphocyte predominant variety. According to prognosis NHL is subdivided into aggressive and indolent lymphoma. Indolent lymphoma has better prognosis with median survival of about 10 years. Follicular lymphoma is the most common indolent lymphoma and diffuse large B-cell lymphoma (DLBCL) is the most aggressive type (2).

Clinical presentation of lymphoma depends on anatomical distribution of disease. Patients usually present with enlarged non tender lymph nodes in HL and two-third of NHL. Remaining one-third of NHL present with symptoms associated with involvement of extra nodal sites like skin, stomach or brain. Treatment strategy of lymphoma depends upon the staging of disease which defines disease location, extension, suggests prognostic information and provides a baseline against which treatment response or disease progression can be compared (3). Accurate staging can minimize the therapeutic toxic effect and reduces the morbidity and mortality associated with therapy. PET-CT is a noninvasive, three-dimensional, metabolic imaging technique consists of PET by 18 F-FDG that can detect glucose metabolism within the cell and low dose CTwhich is used for anatomical location. PET-CT plays a critical role as a baseline measurement before therapy which increases the accuracy of subsequent response assessment. Accurate staging used for pretreatment risk stratification and selection of therapy those ensure fewer patients are undertreated or over treated (4). More than $90 \%$ of patients with newly diagnosed $\mathrm{HL}$ are curable with radiation therapy alone or combination of chemotherapy and radiotherapy (5). Correct staging is important for the selection of appropriate treatment regimen for lymphoma patients.

PET-CT can detect metabolic changes in the areas 
involved with malignant lymphoma before the structural changes become visible (2). In 1999, the National Cancer Institute Working group first published universally accepted guidelines for performing and interpreting PET imaging using ${ }_{18} \mathrm{~F}$ FDG, immunohistochemistry and flow cytometry to see the treatment response criteria for both HL and NHL. These criteria were revised in 2007 by the International Working Group (IWG) (4).International Conference on Malignant Lymphoma (ICML) working group recommended PET$\mathrm{CT}$ as the gold standard for routine staging of FDG avid nodal lymphoma (3).

In Bangladesh, staging of lymphoma is mainly based on conventional staging method. PET-CT is available as regular weekly service since January 2016 at NINMAS. No study has been done yet in Bangladesh to see the extent of variation in staging of both pediatric and adult lymphoma between conventional and PET-CT based staging. This study was designed to analyze the clinical staging and PET-CT based staging in diagnosed lymphoma patient at baseline so that to establish PET-CT based staging in lymphoma patients at baseline in routine use in Bangladesh.

\section{PATIENTS AND METHODS}

This cross sectional observational type of study was carried out at NINMAS, BSMMU campus, Dhaka during the period of July 2017 to June 2018. Newly diagnosed lymphoma patients referred to the institute (NINMAS) for baseline staging were included in this study. A total of 26 patients were enrolled in this study. All the patients were informed of the potential risks and benefit of the procedure and informed consent was taken from each individual case before inclusion in the study. Details of the clinical history, histopathological report and immuno-histochemistry reports were recorded in a structured data sheet. Lymphoma diagnosis was confirmed by histopathology and Immuno-histochemistry reports.

Clinical staging (Ann Arbor staging) was done by clinical history and physical examination. ${ }_{18} \mathrm{~F}-\mathrm{FDG}$ was administered intravenously after 6 hours fasting. To allow the distribution and uptake of radiotracer, the patient was kept in resting quietly for 60 minutes in a shielded room. Imaging was performed with GEDiscoveryTM PET-CT-series 710 with LYSO crystal scanner on an integrated 128-slice CT. Scan was performed from vertex to mid-thigh, 2 minutes per bed usually with 9 bed positions; this varies according to height of the patient. Serum blood glucose level was $<8.0 \mathrm{~m} \mathrm{~mol} / \mathrm{L}$ at the time of injection. SUV is calculated as SUV bw $\mathrm{gm} / \mathrm{ml}$. Images were reviewed in the transaxial, coronal and sagittal planes. High resolution CT scan without contrast was performed for attenuation correction before PET acquisition. Interpretation done by experienced nuclear medicine physician and radiologist. Staging of lymphoma patients were done according to (Figure: 1-4).
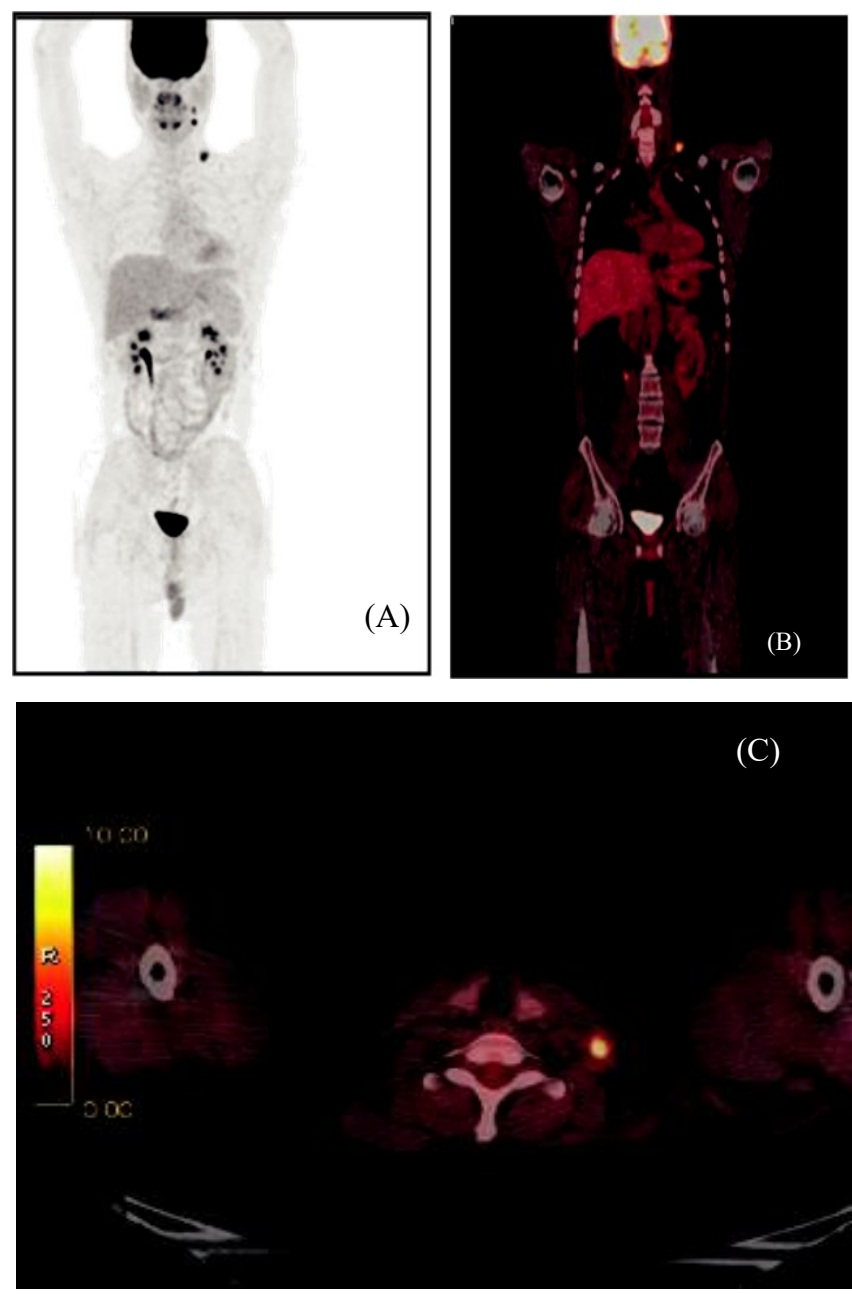

Figure 1: PET-CT scan image of a 24 year male with HL, stage I showing FDG avid lymph node at level $\mathrm{V}$ of left cervical region. A) Maximum intensity projection (MIP) image of whole body PET-CT scan. B) Coronal section of PET-CT fused scan. C) Axial section of PET-CT scan at cervical 


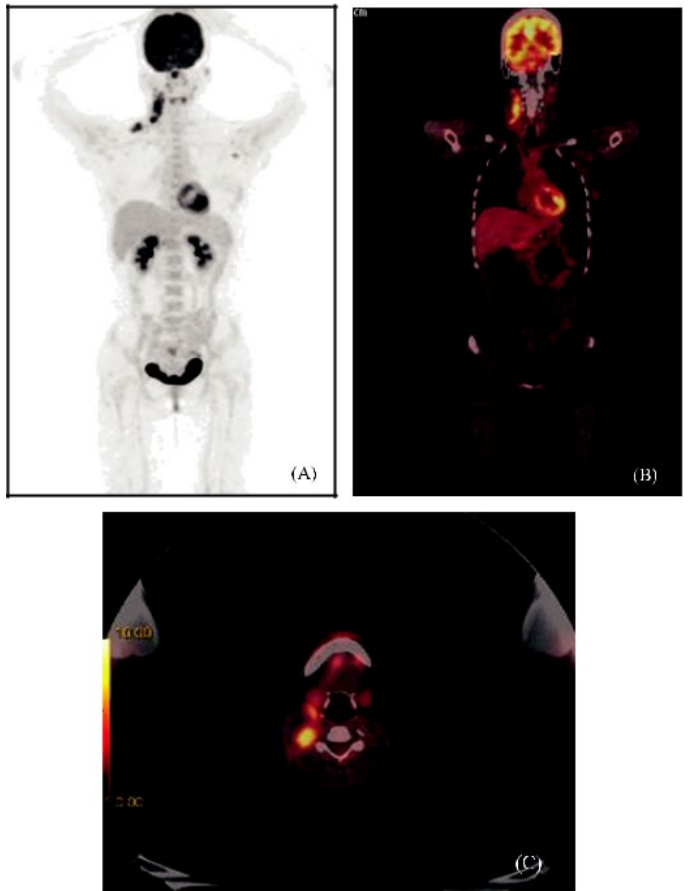

Figure 2: A 35 years female with HL showing FDG avid lymph nodes in cervical and axillary regions (stage-II).

A) Maximum intensity projection (MIP) image of whole body PET-CT scan. B) Coronal section of PET-CT fused scan. C) Axial section of PET-CT scan at cervical region.

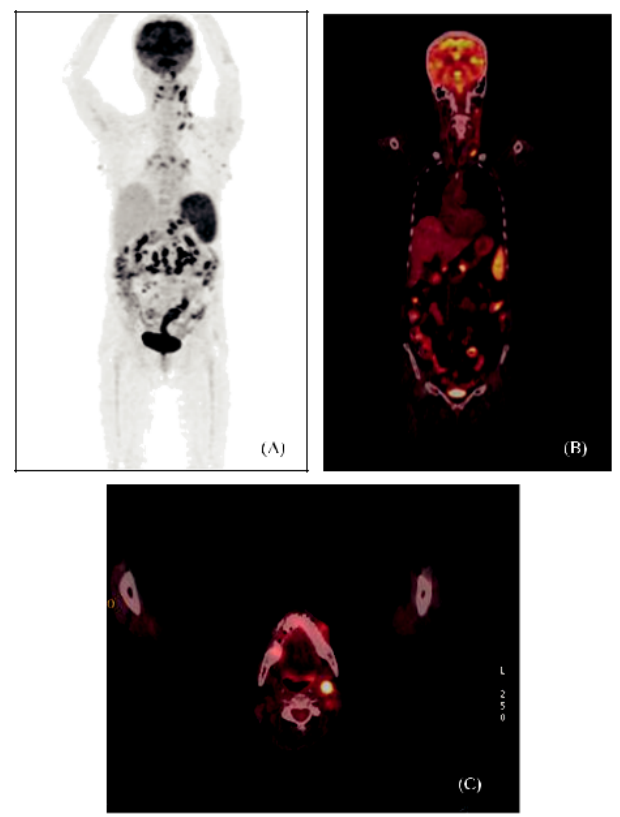

Figure 3: A 65 years female with NHL showing FDG avid lymph nodes in cervical, axillary, abdominal pelvic and inguinal regions (stage-III). A) Maximum intensity projection (MIP) image of whole body PET-CT scan. B) Coronal section of PET-CT fused scan. C) Axial section of PET-CT scan at cervical region.

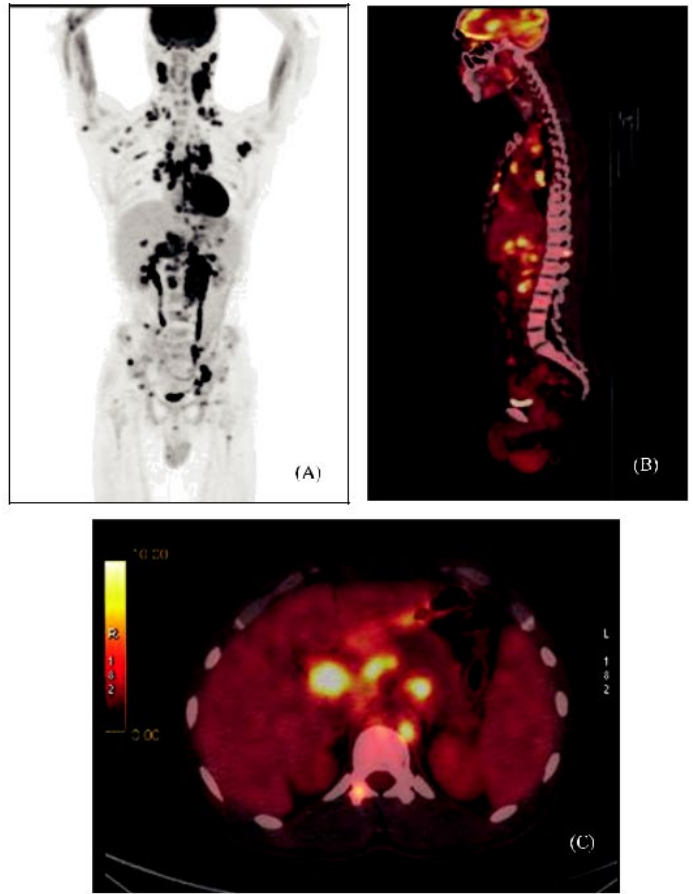

Figure 4: PET-CT scan image of a 25 year male with HL, stage IV showing FDG avid lymph nodes at level above and below the diaphragm with skeletal metastasis (stage-IV). A) Maximum intensity projection (MIP) image of whole body PET-CT scan. B) Saggital section of PET-CT fused scan. C) Axial section of PET-CT scan at lumbar region.

The collected data were compiled and analyzed using window based computer software MS Excel and Statistical Package for Social Sciences (SPSS-23) by appropriate statistical methods. Continuous data were presented as mean and standard deviation (SD). Categorical/qualitative data were presented as percentage (\%).Various tables and figures were used for data presentation.

\section{RESULTS}

Total 26 patients (M-14, F-12) age ranging from 06-68 years (mean $\pm \mathrm{SD}=43.42 \pm 17.98$ years) were included (Figure 5).

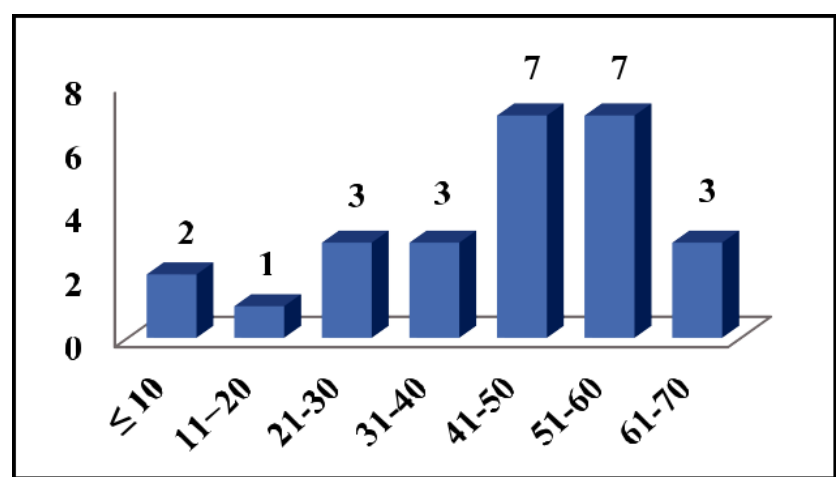

Figure 5: Distribution of the study subjects by age $(n=26)$ 
It was observed that 14 (53.8 \%) patients were Hodgkin Lymphoma and only 12 (46.2\%) were Non Hodgkin Lymphoma (Table1).Out of 14 cases of HL, 5(35\%) patients were presented with classical HL, 4 (28\%) patients were mixed cellularity, 3 (21\%) patients were nodular sclerosis and $2(14 \%)$ patients were lymphocyte rich type of HL. Among 12 cases of NHL, 8 (66.6\%) patients were diffuse large B cell lymphoma (DLBCL), 3 (25\%) patients were follicular lymphoma and $1(8.3 \%)$ patient presented with T-cell lymphoma.

Table 1: Category of lymphoma in study patients

\begin{tabular}{ccc}
\hline Diagnostic status & Frequency & Percentage \\
\hline Hodgkin Lymphoma & 14 & 53.8 \\
Non Hodgkin Lymphoma & 12 & 46.2 \\
Total & $\mathbf{2 6}$ & $\mathbf{1 0 0 . 0}$ \\
\hline
\end{tabular}

Clinical staging status of all the patients was evaluated according to history and physical examination. It was observed that $12(46.2 \%)$ patients were in Stage I, $11(42.3 \%)$ patients were in Stage II and only $3(11.5 \%)$ patients were in Stage III by clinical staging (Table 2).

Table 2: Distribution of patients by clinical staging

\begin{tabular}{ccc}
\hline Clinical Staging & Frequency & Percentage \\
\hline Stage I & 12 & 46.2 \\
Stage II & 11 & 42.3 \\
Stage III & 3 & 11.5 \\
Total & $\mathbf{2 6}$ & $\mathbf{1 0 0 . 0}$ \\
\hline
\end{tabular}

In baseline PET-CT, it was observed that 5(19.2\%) patient were in Stage I, 8 (30.8\%) patient were in Stage II, 6 (23.1\%) patient were in Stage III and 7 (26.9\%) patients were in stage IV by PET-CT staging (Table 3 ).

Table 3: Distribution of patients by PET-CT staging

\begin{tabular}{ccc}
\hline PET-CT Staging & Frequency & Percentage \\
\hline Stage I & 5 & 19.2 \\
Stage II & 8 & 30.8 \\
Stage III & 6 & 23.1 \\
Stage IV & 7 & 26.9 \\
Total & $\mathbf{2 6}$ & $\mathbf{1 0 0 . 0}$ \\
\hline
\end{tabular}

Figure 6 shows out of 26 patients $7(27 \%)$ patients had extranodal involvement out of 26 patients in PET-CT and involved in bone (3 lesions), liver (3 lesions), lung (1 lesion) and spleen (1 lesion).

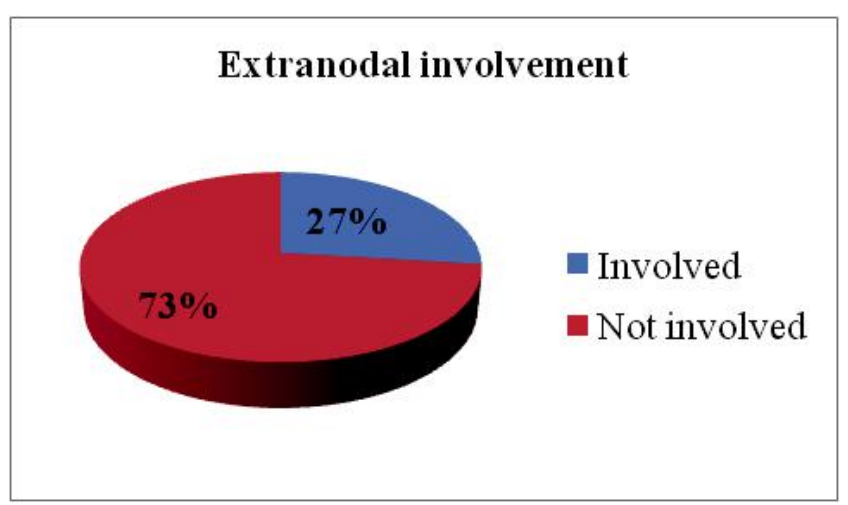

Figure 6: Extranodal involvement in lymphoma patients evaluated by PET-CT.

Table 4 shows key lesions by SUVmax in different stages of lymphoma (stage I, II, III, IV)on PET-CT based staging. Highest SUVmax of key lesion was found in stage 4.

Table 4: Ranges of SUVmax of key lesions on PETCTscan in lymphoma patientsat different stages.

\begin{tabular}{cccc}
\hline Stage & $\begin{array}{c}\text { Lowest } \\
\text { SUVmax }\end{array}$ & $\begin{array}{c}\text { Highest } \\
\text { SUVmax }\end{array}$ & Range \\
\hline Stage I & 7.5 & 9.0 & $7.5-9.0$ \\
Stage II & 9.4 & 11 & $9.4-11$ \\
Stage III & 12.6 & 19 & $12.6-19$ \\
Stage IV & 17.9 & 22.7 & $17.9-22.7$ \\
\hline
\end{tabular}

The comparison between clinical staging and PET-CT based staging was shown in Table 5. It was observed that 53\% patients were upstaged based on PET-CT scan from different stages of clinical staging. There was no down staging.

Table 5: Distribution of patient by Clinical staging vs PET-CT based staging

Clinical Staging PET-CT staging Upstaging

(n) Stage I Stage II Stage III Stage IV (n)

(\%)

\begin{tabular}{cccccccc|}
\hline Stage I & 12 & 5 & 3 & 3 & 1 & 7 & \\
Stage II & 11 & 0 & 5 & 1 & 5 & 6 & $53 \%$ \\
Stage III & 3 & 0 & 0 & 2 & 1 & 1 & \\
Total & 26 & 5 & 8 & 6 & 7 & 14 & \\
\hline
\end{tabular}

\section{DISCUSSION}

Lymphoma is a heterogeneous group of diseases which represents the fifth most common malignancy in the United States (6). HL typically originates in lymphoid 
tissues which tends to develop in an orderly fashion and can spread to other organs. On the other hand, NHL is a heterogeneous group of lymphoproliferative malignances with different patterns of behavior and varying response to treatment (7). More than $90 \%$ of patients with newly diagnosed HL are curable with radiation therapy alone or combination of chemotherapy and radiotherapy (5). Correct staging is important for the selection of appropriate treatment regimen for lymphoma patients.

This observational type of study was carried out with an aim to observe the stage change of clinical staging after doing PET-CT scan and also create awareness about the accurate staging of lymphoma at baseline for the selection of proper treatment strategy.

A total of 26 diagnosed lymphoma patients, by histopathology and Immuno-histochemistry, were included in this study. The study was carried out at NINMAS under BSMMU campus during July 2017 to June 2018.Among them 14 patients were HL and 12 patients were NHL. Pregnancy, lactation, tuberculosis and infectious disease were excluded from the study. The present study findings were discussed and compared with previously published relevant studies.

In this study, it was observed that majority (26.9\%) patients belonged to age 41-50 years and 51-60 year of age. The mean age was found $43.42 \pm 17.98$ years ranged from 06-68 years. Lymphoma can occur at any age, but it is one of the most common causes of cancer in children and young adults aged 15 - 24 years. The risk of HL rises again in late adulthood (after age 55). Overall, the average age at the time of diagnosis is 39 . However, it has a bimodal variation with one peak in early years of life and other after middle age (8).

The incidence of lymphoma is predominant in male than female. The incidence of lymphoma in males was higher in earlier age, but in older ages the incidence of lymphoma in female increases and approaches nearly that of males (8).In this study, it was observed that (54\%) patients were male and $(46 \%)$ were female. Similar findings were noted by Schoder H et al. (2001), they found $56.4 \%$ were male and $43.5 \%$ female in their study subjects (9).Hutchings M et al (2006) also observed male predominance (10).

In this study, it was observed that 14 patients $(53.8 \%)$ were HL and 12 patients (46.2\%) were NHL. NHL is more common than HL. NHL represents nearly $85 \%$ of lymphoma (11). Hingorjo M R and Syed S (2008) found $81.6 \%$ NHL and $18.3 \%$ HL in one study (8). Pelosi E.et al (2008) also found the similar findings (12). Majority of the study showed higher proportion of NHL as compared to HL. Immuno-suppression is the well established risk factor for the development of non-Hodgkin lymphoma. Patients with HIV have an increased risk for high-grade non-Hodgkin lymphoma (7). On the other hand, HL is one of the most frequent lymphomas in the Western world. The annual incidence is about 3 cases per 100,000 persons (13).

Lymphadenopathy was a very common finding on general examination in both NHL and HL patients. In this study, most of the patients presented with cervical (50\%), para-aortic and abdominal (23\%), inguinal (19.2\%) and axillary (11\%) lymphadenopathy. Few of the patients presented with supra-clavicular swelling and swelling of forehead and elbow. Hingorjo M R and Syed S (2008) also found para-aortic, cervical and axillary and inguinal lymphadenopathy as the commonest site for both HL and NHL (8). Mondal, S. K., et al (2013) found Cervical lymph nodes was the most common site (20.7\%) followed by axillary nodes (14.35\%) and the inguinal nodes $(7.9 \%)$, which were similar to the current study (14).If the largest dimension of a node or nodal mass is $10 \mathrm{~cm}$ or greater it is defined as bulky. In this study, no bulky disease was found.

In this study, it was observed that out of 14 cases of HL, $5(35 \%)$ patients were presented with classical HL, $4(28 \%)$ patients were mixed cellularity, $3(21 \%)$ patients were nodular sclerosis and $2(14 \%)$ patients were lymphocyte rich type of HL. Among 12 cases of NHL, $8(66.6 \%)$ patients were diffuse large B cell lymphoma 
(DLBCL), 3(25\%) patients were follicular lymphoma and $1(8.3 \%)$ patient presented with T-cell lymphoma. Mondal, S. K.,et al (2013) found most common sub type diffuse large B cell lymphoma (74.1\%), which are consistent with the current study (14).

Diagnosis of lymphoma patients were done by excision biopsy and histipathological reports which was confirmed by immunohistochemistry analysis then referred to NINMAS for PET-CT scan. A complete clinical evaluation was done by history and physical examination of different groups of lymph nodes, liver and spleen. Clinical staging was done based on Ann Arbor classification for both HL and NHL. Depending on involvement of lymph nodes, they are classified as 4 stages (stage I, II, III, IV). In this study, clinical staging mainly based on physical examination which was compared to PET-CT based staging.

Historically, staging of lymphoma changes time to time and different periods.This changes occur with the invention of different imaging modalities, histopathological examination technique and expansion of clinical knowledge. Formerly, organomegaly was defined by CT for staging of lymphoma. CT scan causes high radiation exposure that limit the routine use. On the other hand, PET-CT is a non-invasive metabolic imaging technique that can detect malignant lymphoma before any visible structural change by detecting metabolic change of tissue or organ (2). PET-CT is more sensitive compare to CT for detection of lymphomatous involvement of normal sized lymph nodes, bone marrow, spleen and extra nodal tissues (15). PET was incorporated in staging and response assessment of lymphoma (both for HL and NHL) by IWG in 2007. Subsequently it was accepted in a workshop which was held 11th ICML in Lugano, Switzerland, in June 2011, which was revised at the12th ICML in 2013.The aims of Lugano classification were to develop universally accepted, improved staging and response criteria for both $\mathrm{HL}$ and NHL.

PET-CT can be used to observe the responses and to guide further therapy in the view of improving treatment outcome. A five-point scale (5-PS) is used for visual assessment to see the degree of response at mid and end of the treatment. The preferable reference scale should be the mediastinum and the liver. This scale was recommended as the standard reporting tool at the First International Workshop on PET in Lymphoma in Deauville, France, in 2009, and these so-called Deauville criteria(16).Deauville criteria is used to see the treatment response based on scoring. It can predict treatment response and categorize disease after chemotherapy and radiotherapy.

In this study, PET-CT scan was done on 26 patients of diagnosed lymphoma to find out any change with clinical staging. Result showed that the PET-CT led to change in the clinical staging in $53 \%$ of patients. No change in the clinical stage was found in $47 \%$ of patients. Three patients, who were prior grouped as stage I on clinical staging, were upstaged to stage II, 3 patients from stage I toIII and another from stage I-IV. One patient was upstaged from stage II to III and 5 patients from stage II to IV. One patient was upstaged from stage III to IV. There was no patient at stage IV on clinical staging but 7 patients were found at stage IV based on PET-CT scan staging (Table 6).

Barrington S F et al. (2016) studied to see the staging assessment for baseline lymphoma. A total of 159 patients (14\%) were upstaged and 74 patients $(6 \%)$ were down staged by PET-CT (17).Pelosi E et al. (2008) evaluated the use of PET-CT for baseline lymphoma patient. Wholebody FDG-PET-CT correctly staged 61 patients out of 65 patients $(93.8 \%)$. PET upstaged 8(12.3\%)patients and downstaged3(4.6\%)patients (12). Schoder H et al. (2001) wanted to see the correct staging for selecting the appropriate treatment for lymphoma patient. The study population consisted of 61 male and 47 female patients. PET led to a change in the clinical stage in $44 \%$ of patients $(n=23)$. A total of $21 \%$ patients were upstaged $(n=11)$ and $23 \%$ patients were down staged (n

$=12$ ). No change in the clinical stage was reported in $52 \%$ of patients (9). Paes F M et al. (2010) found 32\% 
upstaged and 15\% down staged for HL and 31\% upstaged and only $1 \%$ down staged for NHL (15).

Regarding the changes of lymphoma staging at baseline using PET-CT scan in comparison to clinical staging, majority of the study showed that upstaging was more than that of the down staging which are comparable with the current study. Few of the study showed more down staging is more than upstaging. Upstaging was due to FDG avid lymphoma and extranodal involvement. In this present study, it was observed that, there was no down staging based on PET-CT scan, because there was no non FDG avid lymphoma. Presence of at least one focus of ${ }_{18} \mathrm{~F}-\mathrm{FDG}$ uptake is considered as FDG avid. On the other hand, disease proven by clinical examination, conventional imaging modalities, and histopathology with no ${ }_{18} \mathrm{~F}-\mathrm{FDG}$ uptake in any of the involved sites is considered as non FDG avid (18).Avidity of lymphoma correlates with aggressiveness of disease. Low grade or indolent lymphoma is less FDG avid and aggressive lymphoma is more FDG avid (9). Weiler-Sagie et al., (2009) found 94\% FDG avid lymphoma and only 6\% non FDG avid lymphoma (18).

SUV is used as a semi-quantitive measures to express the degree of FDG uptakeand aids in the interpretation of PET scans(3). Key lesion was observed based on SUVmax. In this current study, it was observed that, SUVmax of key lesions were found in stage I ranged from 7.5-9.0, in stage II ranged from 9.4-11, in stage III ranged from 12.6-19 and in stage IV ranged from 17.922.7 (Table 5). Highest SUVmax of key lesion was found in stage IV. One study was done in NINMAS before this study about PET-CT on staging of baseline lymphoma in pediatric age group (19). SUVmax of key lesion was also higher in stage IV ranged 6-35.

Lymphomatous infiltration of any site of body other than lymph node is called extranodal involvement. In this present study, it was observed that, 7(27\%) patients had extranodal involvement out of 26 patients in PETCT scan. Extranodal involvement involved in bone (3 lesions), liver (3 lesions), lung (1 lesion) and spleen (1 lesion).

Exact incidence of lymphoma in Bangladesh is not known. According to WHO, incidence of cancer in Bangladesh were 122,715 patients per year in Bangladesh in 2012. Hematological malignancies are approximately $6.5 \%$ of cancer incidence worldwide. In one study, Hossain M S et al. (2014) found that, lymphoma was about $21 \%$ of all hematological malignancy in Bangladesh (1). PET-CT is costly imaging modality and available in Bangladesh since 2011 in private sector. PET-CT is available as regular weekly service since January 2016 at NINMAS. PET-CT scan facility can cover only few percentages of all lymphoma patients. Previously a large number of patients went abroad for baseline assessment as well as to assess the treatment response. Now, PET-CT is available in our country. So, tendency of patients to go abroad for PET-CT scan has been reduced. Still PET-CT facility should be increased at public hospitals to meet the need.

The limitations of the study are- the study population was selected from one tertiary hospital in Dhaka city, so that the results of the study may not reflect the exact prevalence of lymphoma of the country. The present study was conducted at a very short period of time. Small sample size was also a limitation of the present study. Therefore, in future further study may be under taken with large sample size.

\section{CONCLUSION}

In this study, 14(53\%) patients were upstaged on PETCT scan based staging in comparison to clinical staging. Treatment strategy was planned by baseline staging. PET-CT plays an important role for accurate staging in lymphoma patients at baseline which increases the accuracy of subsequent response assessment. It is recently worldwide established that PET-CT based staging is superior to conventional staging but in Bangladesh staging is mainly based on clinical assessment as this modern facility is only available in 
limited hospitals. From the result of the study, PETCT based staging in lymphoma patients at baseline is recommended for selecting the appropriate treatment regimen and to assess the treatment response.

\section{REFERENCES}

1. Hossain MS, Iqbal MS, Khan MA and et al. Diagnosed hematological malignancies in Bangladesh- a retrospective analysis of over 5000 cases from 10 specialized hospitals. BMC Cancer 2014;14:438. https://doi.org/10.1186/1471-2407-14-438.

2. D'souza MM, Jaimini A, Bansal A. et al. FDG-PET/CT in lymphoma. Indian J Radiol Imaging 2013;23(4):354365.https: //dx.doi.org/10.4103\%2F0971-3026.125626.

3. Biggi A, Guerra L, Hofman MS. Current status of FDG$\mathrm{PET} / \mathrm{CT}$ in staging of adult lymphoma. ClinTransl Imaging 2015;3:253-69. https://doi.org/10.1007/s40336-015-0127-x.

4. Cheson BD, Fisher RI, Barrington SF. et al. Recommendation for Initial Evaluation, Staging, and Response Assessment of Hodgkin and NonHodgkin Lymphoma: The Lugano Classification. J ClinOncol 2014;32(27):3059-67 https://dx.doi.org/10.1200\%2FJCO.2013.54.8800.

5. Canellos GP, Rosenberg SA, Friedberg JW, Lister TA \&DeVita VT. Treatment of Hodgkin lymphoma: a 50-year perspective. J ClinOncol 2014;32(3):163-168.doi/full/10.1200/JCO.2013.53.1194.

6. JemalA, Siegel R, Ward E. et al. Cancer statistics. CA: a Cancer Journal for Clinician 2008;58 (2): 71-96. https://doi.org/ 10.3322/ CA.2007.0010.

7. Shankland KR, Armitage JO \& Hancock BW. Non-hodgkin lymphoma. The Lancet 2012;380(9844): 848-857. https://doi.org/ 10.1016/S0140-6736(12)60605-9.

8. Hingorjo MR \& Syed S. PRESENTATION, STAGING AND DIAGNOSIS OF LYMPHOMA: A CLINICAL PERSPECTIVE. J Ayub Med Coll Abbottabad 2008; 20(4).

9. Schoder H, Meta J, Yap C, Ariannejad M, Rao J, Phelps ME, Peter E, Sayre VJ \&Czernin, J. Effect of Whole-Body 18F -FDG PET Imaging on Clinical Staging and Management of Patients with Malignant Lymphoma. JNM 2001;42(4):1139-1143.
10. Hutchings M, Loft A, Hansen M, Pedersen LM, Berthelsen AK, eiding S, D'Amore F, Boesen A-M, Roemer L \& Specht L. Positron emission tomography with or without computed tomography in the primary staging of Hodgkin's lymphoma. Malignant Lymphomas2006;91(4):482-489.

11. Lu P. Staging and classification of lymphoma.In Seminars in nuclear medicine2005;35(3):160-164.https://doi.org/10.1053/j.semnuclmed.2005.02 .002 .

12. Pelosi E, Pregno P, Penna D, et al. Role of whole-body [18F] fluorodeoxyglucose positron emission tomography/computed tomography (FDG-PET/CT) and conventional techniques in the staging of patients with Hodgkin and aggressive non Hodgkin lymphoma.Radiol Med 2008;113:578-590.https: //doi.org/10.1016/ j.clinimag.2008.10.023.

13. Küppers R, Engert A \&Hansmann ML. Hodgkin lymphoma. JCI 2012;122(10):3439-3447.https://doi.org/10.1172/JCI61245.

14. Mondal SK, Mandal PK, Samanta TK, Chakaborty S, Roy SD \& Roy S. A retrospective analysis of 455 cases according to World Health Organization classification. Indian J Med Paediatr Oncol2013; 34:242246. https://dx.doi.org/10.4103\%2F0971-5851.125235.

15. Paes FM, Kaikanis DG, Siders PA and Serafini AN. FDG $\mathrm{PET} / \mathrm{CT}$ of Extranodal Involvement in Non-Hodgkin Lymphoma and Hodgkih Disease. RadioGraphics2010; 30:269-291.https: //doi.org/10.1148/ rg.301095088.

16. Meignan M, Galamini A, Haioun C. et al. Report on the First International Workshop on interim-PET scan in lymphoma. Leukemia \& Lymphoma August 2009;50(8): 1257-60.https: //doi.org/ 10.1080/10428190903040048.

17. Barrington SF, Kirkwood AA, Franceschetto A. et al. PET-CT for staging and early response: results from the Response-Adapted Therapy in Advanced Hodgkin Lymphoma study. Blood 24 March 2016;127(12):1531-38.doi: https://doi.org/10.1182/ blood-2015-11-679407.

18. Weiler-Sagie M, Bushelev O, Epelbaum R, Dann EJ, Haim N, Avivi I, Ben-Barak A, Arie-Ben Y. Bar-Shalom R. \& Israel O. 18F-FDG Avidity in Lymphoma Readdressed: A Study of 766 Patients. JNM 2009;51:25-30.doi:10.2967/jnumed.109.067892.

19. Begum F, Nisa L, Quadir KA, Begum SMF, Hussain R, Rahman ATM A, Khan ZJ, Siddique MAB, Pascual, T. \& Hasan M. Evaluation of F-18 PET-CT in Pediatric Lymphoma- Preliminary Experience at National Institute of Nuclear Medicine and Allied Sciences. Asia-Ocean J Nucl Med Biol 2016;4(1):S51. 